\title{
Gauge fields on noncommutative geometries with curvature
}

\author{
M. Burić, ${ }^{a}$ H. Grosse ${ }^{b}$ and J. Madore ${ }^{c}$ \\ ${ }^{a}$ Faculty of Physics, University of Belgrade, \\ Studentski trg 12, SR-11001 Belgrade, Serbia \\ ${ }^{b}$ Department of Physics, University of Vienna, \\ Boltzmanngasse 5, A-190 Vienna, Austria \\ ${ }^{c}$ Laboratoire de Physique Théorique, Université de Paris-Sud, \\ Bâtiment 211, F-91405 Orsay, France \\ E-mail: majab@phy.bg.ac.rs, harald.grosse@univie.ac.at, \\ madore@th.u-psud.fr
}

ABSTRACT: It was shown recently that the lagrangian of the Grosse-Wulkenhaar model can be written as lagrangian of the scalar field propagating in a curved noncommutative space. In this interpretation, renormalizability of the model is related to the interaction with the background curvature which introduces explicit coordinate dependence in the action. In this paper we construct the $U_{1}$ gauge field on the same noncommutative space: since covariant derivatives contain coordinates, the Yang-Mills action is again coordinate dependent. To obtain a two-dimensional model we reduce to a subspace, which results in splitting of the degrees of freedom into a gauge and a scalar. We define the gauge fixing and show the BRST invariance of the quantum action.

Keywords: Non-Commutative Geometry, Gauge Symmetry, Renormalization Regularization and Renormalons

ARXIV EPRINT: 1003.2284 


\section{Contents}

$\begin{array}{llr}1 & \text { Introduction } & 1\end{array}$

2 Truncated Heisenberg algebra $\quad 2$

$\begin{array}{lll}3 & \text { Gauge fields } & 7\end{array}$

4 Yang-Mills action $\quad 9$

5 Chern-Simons action $\quad 11$

$\begin{array}{llr}6 & \text { BRST invariance } & 12\end{array}$

$\begin{array}{lll}7 & \text { Conclusions } & 13\end{array}$

\section{Introduction}

Gauge theories were first formulated on matrix geometries late in the last century [1-3]. At first interest was concentrated on their properties as classical field theories and mainly based on the fact that the extension $\grave{a}$ la Kaluza-Klein of an ordinary geometry by an algebra of $n \times n$ matrices transforms electromagnetism, a $U_{1}$-theory, into a Yang-MillsHiggs-Kibble $(S) U_{n}$ theory. Subsequent to the work of Seiberg and Witten at the dawn of the new century [4] the interest of the majority of workers in the field focused on Moyal spaces $[5,6]$, partly because of the unique algorithm which gives the description of field theories. It was soon realized that upon quantization the gauge fields are plagued with the same $U V / I R$-mixing behavior as the scalar field, that is, although the resulting models remain as they should $U V$-finite, the noncommutativity gives rise to a new $I R$ divergence.

This renormalizability problem has been recently solved in the case of scalar field by Grosse and Wulkenhaar (GW) by including an additional term of the form $x^{2} \phi^{2}$ in the action, [7-9]. The oscillator term provides the symmetry between short and long distances or between coordinates and momenta which is referred to as Langmann-Szabo (LS) duality, [10]. The external potential in the GW action breaks translational invariance; scalar models which are translationally invariant and LS dual were subsequently proposed and proved to be renormalizable, $[11,12]$. They generically contain an additional term $\partial^{-2} \phi^{2}$ in the action which changes the form of the scalar field propagator to $\left(p^{2}+m^{2}+\frac{a}{p^{2}}\right)^{-1}$.

One would like to be able to construct renormalizable models for the gauge fields in a similar way. However, there is a problem which has not been satisfactorily solved: how to include the confining coordinate-dependent terms in covariant or systematic manner. There are various proposals which we recall briefly here, for more detailed reviews see [13, 14]. Possibility which the authors of $[14,15]$ took is to start from a scalar field interacting with 
an external gauge field, and then integrate the scalar degree of freedom. In this manner they obtained the 'induced gauge model': it is expressed in terms of the so-called covariant coordinates and includes thus spatial coordinates in a natural way. However the model does not have a trivial vacuum so it is not clear how to quantize, $[16,17]$. The other possibility to introduce coordinate dependence is through the ghost sector: such a model was described in [18], where also its BRST invariance was proved. The $\partial^{-2}$ gauge theories were defined and explored in considerable details in [19]. However a full renormalizability analysis of mentioned models for various reasons is still missing.

In recent paper [20] we proposed a geometric interpretation of the oscillator term: It was shown there that the Grosse-Wulkenhaar action can be interpreted as an action for the scalar field coupled to the curvature of a background noncommutative space. The coupling has the usual form, $R \phi^{2}$, and in this term the oscillator potential is contained. In this paper we explore further the differential structure of the mentioned background space (which we call the truncated Heisenberg algebra) and we construct the $U_{1}$ gauge theory on it: the resulting action presents geometric analog of the GW action for the gauge fields. We also initiate the study of renormalizability by proving the BRST invariance of the gauge fixed action.

The plan of the paper is the following: in section 2 we recollect some results of [20] and also some steps of the construction of local symmetries in the noncommutative frame formalism, $[1-3,21]$. We apply the formalism to the truncated Heisenberg space and then we reduce to subspace $z=0$ which gives the relevant two-dimensional theory in section 3. In sections 4 and 5 we discuss the Yang-Mills and the Chern-Simons actions and the corresponding classical equations of motion. Finally in the last section we specify the gauge fixing and we show that the quantum action is BRST invariant. Note that, in notation which we use, coordinates and their functions (fields) are generically operators and therefore the product is always noncommutative: depending on the representation it is either matrix product or the Moyal product. Similarly the trace denotes respectively the matrix trace or the integral.

\section{Truncated Heisenberg algebra}

Generically a noncommutative space is an algebra $\mathcal{A}$ generated by a set of hermitian elements which we shall loosely refer to as 'coordinates'. The truncated Heisenberg space, [20], is a three-dimensional noncommutative space defined by coordinates $x, y$ and $z$ and the commutation relations

$$
\begin{aligned}
& {[x, y]=i \epsilon \mu^{-2}(1-\mu z),} \\
& {[x, z]=i \epsilon(y z+z y)} \\
& {[y, z]=-i \epsilon(x z+z x) .}
\end{aligned}
$$

The $\mu$ is a constant of dimension of the inverse length; physically in fact it would make sense (and be consistent) to introduce two different length scales, $\mu$ and $\bar{\mu}$, in (2.1): $\mu$ as a characteristic dimension of $x-y$ space, and $\bar{\mu}$ for the auxilliary $z$-direction. For simplicity however we keep $\mu$ and $\bar{\mu}$ the same. The $\epsilon$ is a dimensionless parameter which indicates 
the relative strength of noncommutativity; we denote $k=\epsilon \mu^{-2}$. For $\epsilon=1$ algebra (2.1) can be represented by $n \times n$ matrices for any integer $n$,

$$
\begin{gathered}
x=\frac{1}{\mu \sqrt{2}}\left(\begin{array}{cccccc}
0 & 1 & 0 & \cdot & \cdot & \cdot \\
1 & 0 & \sqrt{2} & \cdot & \cdot & \cdot \\
0 & \sqrt{2} & 0 & \cdot & \cdot & \cdot \\
\cdot & \cdot & \cdot & \cdot & \cdot & \cdot \\
\cdot & \cdot & \cdot & \cdot & 0 & \sqrt{n-1} \\
\cdot & \cdot & \cdot & \cdot & \sqrt{n-1} & 0
\end{array}\right), \\
y=\frac{i}{\mu \sqrt{2}}\left(\begin{array}{cccccc}
0 & -1 & 0 & \cdot & \cdot & \cdot \\
1 & 0 & -\sqrt{2} & \cdot & \cdot & \cdot \\
0 & \sqrt{2} & 0 & \cdot & \cdot & \cdot \\
\cdot & \cdot & \cdot & \cdot & \cdot & \cdot \\
\cdot & \cdot & \cdot & \cdot & 0 & -\sqrt{n-1} \\
\cdot & \cdot & \cdot & \cdot & \sqrt{n-1} & 0
\end{array}\right),
\end{gathered}
$$

and

$$
z=\frac{n}{\mu}\left(\begin{array}{lllll}
0 & 0 & 0 & \cdots & . \\
0 & 0 & 0 & \ldots & . \\
0 & 0 & 0 & \ldots & . \\
\cdots & \cdots & \cdots & . & . \\
\cdots & \cdots & 0 & 0 & 0 \\
. & . & . & 0 & 1
\end{array}\right)
$$

This is the representation which we shall keep in mind because it is not at the moment clear what are representations of (2.1) for values of $\epsilon$ between $\epsilon=0$ and $\epsilon=1$. Matrices $x$ and $y$ are easily recognizable as quantum-mechanical coordinate and momentum represented in the Fock basis, of course for $n \rightarrow \infty$. The limit can be consistently imposed at the level of algebra as projection to the hyperplane $z=0$. Then the commutation relations reduce to

$$
[x, y]=i \hbar .
$$

Some geometric properties of (2.1) were analyzed in [20]; here we wish to define gauge fields and therefore we need to explore structure of the algebra of differential forms. We have established already that though algebra (2.5), defined as a limit of matrix truncations for $n \rightarrow \infty$, is two-dimensional its cotangent space is three-dimensional. In consequence the corresponding geometry differs from the usual flat geometry of the Moyal space. The same property is shared by differential calculi on other noncommutative spaces, for example on the fuzzy sphere or on the $\kappa$-Minkowski space, [22-24]. Let us first construct the exterior algebra on the full three-dimensional algebra (2.1).

In the approach which we are using the space of 1 -forms $\Omega^{1}$ is spanned by a preferred set of forms $\theta^{\alpha}$ which is called the frame, [21]. Dual to $\theta^{\alpha}$ are the derivations $e_{\alpha}$ as $\theta^{\alpha}\left(e_{\beta}\right)=\delta_{\beta}^{\alpha}$. Differential $d$ of a function $f$ is defined as $d f=e_{\alpha} f \theta^{\alpha}$. The derivations are inner, generated by momenta $p_{\alpha} \in \mathcal{A}, \quad e_{\alpha} f=\left[p_{\alpha}, f\right]$. We take $p_{\alpha}$ to be antihermitian, ${ }^{1}$

\footnotetext{
${ }^{1}$ The $*$-structure is the ordinary one: $p_{\alpha}^{*}$ is the adjoint of the matrix $p_{\alpha}$ in the matrix representation and the adjoint of the corresponding operator in the Moyal space.
} 
so for real $f, e_{\alpha} f$ is real too. Since the differential calculus is defined by $p_{\alpha}$ it is quite clear that in some respect momenta are more fundamental than coordinates. They are also more elementary in the sense that formulae look simpler when expressed in terms of the momenta. For example, it can be shown that $p_{\alpha}$ always form a quadratic algebra

$$
2 P^{\gamma \delta}{ }_{\alpha \beta} p_{\gamma} p_{\delta}-F^{\gamma}{ }_{\alpha \beta} p_{\gamma}-\frac{1}{i \epsilon} K_{\alpha \beta}=0,
$$

that is that commutators between momenta cannot have arbitrary form as can commutators between coordinates. Constants $P^{\gamma \delta}{ }_{\alpha \beta}, F^{\gamma}{ }_{\alpha \beta}$ and $K_{\alpha \beta}$ are called the structure elements; defining

$$
P_{\alpha \beta}^{\gamma \delta}=\frac{1}{2}\left(\delta_{\alpha}^{\gamma} \delta_{\beta}^{\delta}-\delta_{\beta}^{\gamma} \delta_{\alpha}^{\delta}\right)+i \epsilon Q_{\alpha \beta}^{\gamma \delta},
$$

we can rewrite $(2.6)$ as

$$
\left[p_{\alpha}, p_{\beta}\right]=\frac{1}{i \epsilon} K_{\alpha \beta}+F_{\alpha \beta}^{\gamma} p_{\gamma}-2 i \epsilon Q_{\alpha \beta}^{\gamma \delta} p_{\gamma} p_{\delta} .
$$

The choice of the momenta is equivalent to the choice of differential calculus and in principle it is not completely fixed. For the truncated Heisenberg space we have [20]

$$
\epsilon p_{1}=i \mu^{2} y, \quad \epsilon p_{2}=-i \mu^{2} x, \quad \epsilon p_{3}=i \mu\left(\mu z-\frac{1}{2}\right)
$$

and the momentum algebra is given by

$$
\begin{aligned}
& {\left[p_{1}, p_{2}\right]=\frac{\mu^{2}}{2 i \epsilon}+\mu p_{3},} \\
& {\left[p_{2}, p_{3}\right]=\mu p_{1}-i \epsilon\left(p_{1} p_{3}+p_{3} p_{1}\right)} \\
& {\left[p_{3}, p_{1}\right]=\mu p_{2}-i \epsilon\left(p_{2} p_{3}+p_{3} p_{2}\right) .}
\end{aligned}
$$

One can observe that it has neither the structure of a Lie algebra nor the structure of a quantum group. We identify the structure elements

$$
K_{12}=\frac{\mu^{2}}{2}, \quad F_{23}^{1}=\mu, \quad Q^{13}{ }_{23}=\frac{1}{2}, \quad Q^{23}{ }_{31}=\frac{1}{2} .
$$

Since the algebra and the 1-forms commute the algebra of forms is the tensor product $\Omega^{*}(\mathcal{A})=\mathcal{A} \otimes \Lambda^{*}$ of the algebra $\mathcal{A}$ and a finite-dimensional exterior algebra $\Lambda^{*}$ generated by three elements. Obviously the exterior multplication has to be consistent if not completely defined by the differential. In the frame formalism coefficients $P^{\gamma \delta}{ }_{\alpha \beta}$ define the exterior multiplication of two 1-forms $\theta^{\alpha}$ :

$$
\theta^{\gamma} \theta^{\delta}=P_{\alpha \beta}^{\gamma \delta} \theta^{\alpha} \otimes \theta^{\beta}
$$

that is

$$
\theta^{\gamma} \theta^{\delta}+\theta^{\delta} \theta^{\gamma}=2 i \epsilon Q^{\gamma \delta}{ }_{\alpha \beta} \theta^{\alpha} \theta^{\beta}
$$

1-forms $\theta^{\alpha}$ do not anticommute only when the momentum algebra has quadratic terms, $Q^{\gamma \delta}{ }_{\alpha \beta} \neq 0 . P_{\gamma \delta}^{\alpha \beta}$ has to be a projector

$$
P_{\gamma \delta}^{\alpha \beta} P_{\eta \zeta}^{\gamma \delta}=P_{\eta \zeta}^{\alpha \beta},
$$


and also

$$
K_{\alpha \beta} P_{\gamma \delta}^{\alpha \beta}=K_{\gamma \delta}, \quad F_{\alpha \beta}^{\eta} P_{\gamma \delta}^{\alpha \beta}=F_{\gamma \delta}^{\eta}, \quad Q_{\alpha \beta}^{\eta \zeta} P_{\gamma \delta}^{\alpha \beta}=Q_{\gamma \delta}^{\eta \zeta} .
$$

Hermiticity of the basis $\theta^{\alpha}$ can be assumed as in commutative case. Imposing hermiticity on the exterior product implies the condition, [25],

$$
\left(P_{\gamma \delta}^{\alpha \beta}\right)^{*} P_{\eta \zeta}^{\delta \gamma}=P^{\beta \alpha}{ }_{\eta \zeta} .
$$

As it can be checked, formulae (2.14) and (2.15) are on the truncated Heisenberg space satisfied for all values of $\epsilon$, whereas (2.16) is true for $\epsilon=1$.

The $P^{\gamma \delta}{ }_{\alpha \beta} \theta^{\alpha} \theta^{\beta}$ form a basis of the space of 2 -forms $\Omega^{2}$. Anticommutation relations for truncated Heisenberg geometry are given by

$$
\begin{aligned}
\left(\theta^{1}\right)^{2} & =0, \quad\left(\theta^{2}\right)^{2}=0, \quad\left(\theta^{3}\right)^{2}=0, \\
\left\{\theta^{1}, \theta^{2}\right\} & =0, \\
\left\{\theta^{1}, \theta^{3}\right\} & =i \epsilon\left(\theta^{2} \theta^{3}-\theta^{3} \theta^{2}\right), \\
\left\{\theta^{2}, \theta^{3}\right\} & =i \epsilon\left(\theta^{3} \theta^{1}-\theta^{1} \theta^{3}\right),
\end{aligned}
$$

while the canonical basis is

$$
\begin{aligned}
P_{\gamma \delta}^{12} \theta^{\gamma} \theta^{\delta} & =\frac{1}{2}\left[\theta^{1}, \theta^{2}\right], \\
P^{13}{ }_{\gamma \delta} \theta^{\gamma} \theta^{\delta} & =\frac{1}{2}\left[\theta^{1}, \theta^{3}\right]+\frac{1}{2} i \epsilon\left[\theta^{2}, \theta^{3}\right], \\
P^{23}{ }_{\gamma \delta} \theta^{\gamma} \theta^{\delta} & =\frac{1}{2}\left[\theta^{2}, \theta^{3}\right]-\frac{1}{2} i \epsilon\left[\theta^{1}, \theta^{3}\right] .
\end{aligned}
$$

We see that as a basis we can alternatively use the set of anticommutators as in commutative geometry. In fact, the structure of exterior algebra is almost completely customary, at least regarding dimensionalities: spaces $\Omega^{1}$ and $\Omega^{2}$ are three-dimensional, while $\Omega^{0}$ and $\Omega^{3}$ are one-dimensional.

Exterior multiplication can be extended to the product of three 1-forms, but an additional constraint has to be fulfilled. A necessary condition to define the product uniquely is

$$
\mathcal{C}^{\alpha \beta \gamma}{ }_{\eta \zeta \xi} \theta^{\eta} \theta^{\zeta} \theta^{\xi}=0,
$$

where $\mathcal{C}^{\alpha \beta \gamma}{ }_{\eta \zeta \xi}=\mathcal{C}^{\beta \gamma}{ }_{\delta \epsilon} \mathcal{C}^{\alpha \delta}{ }_{\eta \lambda} \mathcal{C}^{\lambda \epsilon}{ }_{\zeta \xi}-\mathcal{C}^{\alpha \beta}{ }_{\delta \epsilon} \mathcal{C}^{\epsilon \gamma}{ }_{\lambda \xi} \mathcal{C}^{\delta \lambda}{ }_{\eta \zeta}$ and constants

$$
\mathcal{C}^{\gamma \delta}{ }_{\alpha \beta}=\delta_{\alpha}^{\gamma} \delta_{\beta}^{\delta}-2 P^{\gamma \delta}{ }_{\alpha \beta}
$$

define the operation which reverses the order of indices in the exterior product,

$$
\theta^{\alpha} \theta^{\beta}=-\mathcal{C}^{\alpha \beta}{ }_{\gamma \delta} \theta^{\gamma} \theta^{\delta} .
$$

Equation (2.19) is a weak form of the braid relation and it is satisfied in our case. This means that the product of three 1 -forms can be defined unambiguously. ${ }^{2}$

\footnotetext{
${ }^{2}$ Relations like (2.14), (2.16), (2.19) which include structure elements were checked using Mathematica.
} 
What one needs in calculation is the twisted-antisymmetric tensor, a generalization of the usual $\delta_{\zeta \eta \xi}^{\alpha \beta \gamma}$ (which in three dimensions reduces to the product, $\delta_{\zeta \eta \xi}^{\alpha \beta \gamma}=\epsilon^{\alpha \beta \gamma} \epsilon_{\zeta \eta \xi}$ ). The neeed generalization is given by

$$
\Delta_{\zeta \eta \xi}^{\alpha \beta \gamma}=\frac{1}{3}\left(-\delta_{\zeta}^{\alpha} P^{\beta \gamma}{ }_{\eta \xi}-\mathcal{C}^{\alpha \beta}{ }_{\zeta \rho} P^{\rho \gamma}{ }_{\eta \xi}+\mathcal{C}^{\beta \gamma}{ }_{\rho \sigma} \mathcal{C}^{\alpha \rho}{ }_{\zeta \tau} P^{\tau \sigma}{ }_{\eta \xi}\right)
$$

Unlike $\delta_{\zeta \eta \xi}^{\alpha \beta \gamma}$, noncommutative $\Delta_{\zeta \eta \xi}^{\alpha \beta \gamma}$ is not a projector; however on the product of three 1 -forms it acts as one,

$$
\Delta_{\zeta \eta \xi}^{\alpha \beta \gamma} \theta^{\zeta} \theta^{\eta} \theta^{\xi}=\theta^{\alpha} \theta^{\beta} \theta^{\gamma} .
$$

We shall need $\Delta_{\zeta \eta \xi}^{\alpha \beta \gamma}$ to define the volume 3 -form $\Theta$, and later on, the action. By definition the integral of a 3 -form $\alpha=f \Theta$ is given by $\int \alpha=\operatorname{Tr} f$.

It is not difficult to write the algebra of 3 -forms on truncated Heisenberg space explicitly. From (2.17) and the associativity of the exterior product we obtain

$$
\begin{aligned}
& \theta^{1} \theta^{3} \theta^{1}=\theta^{2} \theta^{3} \theta^{2} \\
& \theta^{1} \theta^{2} \theta^{3}=-\theta^{2} \theta^{1} \theta^{3}=\theta^{3} \theta^{1} \theta^{2}=-\theta^{3} \theta^{2} \theta^{1}=i \frac{\epsilon^{2}-1}{2 \epsilon} \theta^{2} \theta^{3} \theta^{2}, \\
& \theta^{1} \theta^{3} \theta^{2}=-\theta^{2} \theta^{3} \theta^{1}=i \frac{\epsilon^{2}+1}{2 \epsilon} \theta^{2} \theta^{3} \theta^{2} . \\
& \theta^{3} \theta^{1} \theta^{3}=0, \quad \theta^{3} \theta^{2} \theta^{3}=0 .
\end{aligned}
$$

There is obviously only one independent 3 -form, that is the volume form is unique. We define it as

$$
\Theta=-\frac{i}{2 \epsilon} \theta^{2} \theta^{3} \theta^{2}
$$

in order that it reduce to $\theta^{1} \theta^{2} \theta^{3}$ in the commutative limit. Note that the product of three 1 -forms is not cyclic, for example $\theta^{1} \theta^{3} \theta^{2} \neq \theta^{3} \theta^{2} \theta^{1}$. Relations (2.24) can be rewritten as

$$
\begin{aligned}
& {\left[\theta^{1}, \theta^{2}\right] \theta^{3}=\theta^{3}\left[\theta^{1}, \theta^{2}\right]=2\left(1-\epsilon^{2}\right) \Theta,} \\
& {\left[\theta^{2}, \theta^{3}\right] \theta^{1}=\theta^{1}\left[\theta^{2}, \theta^{3}\right]=2 \Theta} \\
& {\left[\theta^{2}, \theta^{3}\right] \theta^{2}=-\theta^{2}\left[\theta^{2}, \theta^{3}\right]=2 i \epsilon \Theta} \\
& {\left[\theta^{3}, \theta^{1}\right] \theta^{2}=\theta^{2}\left[\theta^{3}, \theta^{1}\right]=2 \Theta} \\
& {\left[\theta^{3}, \theta^{1}\right] \theta^{1}=-\theta^{1}\left[\theta^{3}, \theta^{1}\right]=-2 i \epsilon \Theta .}
\end{aligned}
$$

From (2.24) we see that the value $\epsilon=1$ is special: it gives for example $\theta^{1} \theta^{2} \theta^{3}=0$, which is in the commutative case unusual. Nonetheless the algebra of 3 -forms is nondegenerate with nonvanishing elements $\theta^{1} \theta^{3} \theta^{1}, \theta^{2} \theta^{3} \theta^{2}, \theta^{1} \theta^{3} \theta^{2}$ and $\theta^{2} \theta^{3} \theta^{1}$. As we have mentioned value $\epsilon=1$ is important from the point of view of representations. Therefore we wish to include it explicitly, so we define the Hodge-dual as

$$
*\left(\frac{1}{2}\left[\theta^{1}, \theta^{2}\right]\right)=\theta^{3}, \quad *\left(\frac{1}{2}\left[\theta^{2}, \theta^{3}\right]\right)=\theta^{1}, \quad *\left(\frac{1}{2}\left[\theta^{3}, \theta^{1}\right]\right)=\theta^{2} .
$$

This definition modifies the usual normalization, giving for example

$$
*\left(\frac{1}{2}\left[\theta^{1}, \theta^{2}\right]\right) \frac{1}{2}\left[\theta^{1}, \theta^{2}\right]=\left(1-\epsilon^{2}\right) \Theta .
$$


This implies in particular that for $\epsilon=1$ the 2-form $\left[\theta^{1}, \theta^{2}\right]$ does not have a Hodge dual, and consequently the corresponding term will be absent from the Yang-Mills lagrangian. Note that relations (2.27) give the Hodge dual uniquely only if, when multiplying forms with their duals the products are symmetrized.

Geometric characteristics of the truncated Heisenberg space as the connection and the curvature were discussed in [20]. In order to define gauge fields we only need the Ricci rotation coefficients $C^{\alpha}{ }_{\beta \gamma}, d \theta^{\alpha}=-\frac{1}{2} C^{\alpha}{ }_{\beta \gamma} \theta^{\beta} \theta^{\gamma}$. They are determined by the structure elements,

$$
C_{\alpha \beta}^{\gamma}=F_{\alpha \beta}^{\gamma}-4 i \epsilon Q_{\alpha \beta}^{\gamma \delta} p_{\delta}
$$

For the truncated Heisenberg space we have

$$
\begin{aligned}
& C^{1}{ }_{23}=-C^{1}{ }_{32}=2 \mu^{2} z, \\
& C^{3}{ }_{13}=-C^{3}{ }_{31}=2 \mu^{2} x,
\end{aligned}
$$

\section{Gauge fields}

As mentioned already, to define gauge symmetries we use representation-independent formulation of [1-3] which we will slightly generalize. The focus will be mostly on formulae and their application to the truncated Heisenberg geometry; for more mathematical recent reviews see [14] or [26]. The gauge potential $A$ and the field strength $F$ are respectively a 1-form and a 2-form,

$$
A=A_{\alpha} \theta^{\alpha}, \quad F=d A+A^{2}=\frac{1}{2} F_{\alpha \beta} \theta^{\alpha} \theta^{\beta} .
$$

They are antihermitian and dimensionless; of course, $A$ and $F$ are functions of noncommutative coordinates. As the gauge group we take noncommutative $U_{1}$, the group of all unitary elements of $\mathcal{A}$; the group elements are denoted by $g$. In the finite-matrix representation $U_{1}$ consists of all unitary $n \times n$ matrices and thus, as the set of elements with group multiplication, the noncommutative $U_{1}$ is equal to the usual $U_{n}$ which acts on fields on a commutative space.

$A$ is a connection so it transforms as $A^{\prime}=g^{-1} A g+g^{-1} d g$, that is

$$
A_{\alpha}^{\prime}=g^{-1} A_{\alpha} g+g^{-1} e_{\alpha} g \text {. }
$$

The field strength transforms in the adjoint representation, $F^{\prime}=g^{-1} F g . F$ is a 2-form so it can be expanded in the basis (2.12). From (2.14) we see that components $F_{\alpha \beta}$ satisfy

$$
F_{\zeta \eta}=F_{\alpha \beta} P^{\alpha \beta}{ }_{\zeta \eta}
$$

This means that the components of the field strength are in our case antisymmetric, because coefficients $P^{\alpha \beta}{ }_{\zeta \eta}$ are antisymmetric in the lower pair of indices. From definition (3.1) we obtain

$$
d A+A^{2}=\left(e_{\beta} A_{\gamma}-\frac{1}{2} A_{\alpha} C_{\beta \gamma}^{\alpha}+A_{\beta} A_{\gamma}\right) P^{\beta \gamma}{ }_{\zeta \eta} \theta^{\zeta} \theta^{\eta}
$$


and the field strength is given by

$$
F_{\zeta \eta}=e_{[\zeta} A_{\eta]}-A_{\alpha} C^{\alpha}{ }_{\zeta \eta}+\left[A_{\zeta}, A_{\eta}\right]+2 i \epsilon\left(e_{\beta} A_{\gamma}\right) Q^{\beta \gamma}{ }_{\zeta \eta}+2 i \epsilon A_{\beta} A_{\gamma} Q^{\beta \gamma}{ }_{\zeta \eta} .
$$

In case of vanishing torsion, that is when $\omega^{\alpha}{ }_{[\beta \gamma]}=C^{\alpha}{ }_{\beta \gamma}$, (3.5) can be written as

$$
F_{\zeta \eta}=\nabla_{[\zeta} A_{\eta]}+\left[A_{\zeta}, A_{\eta}\right]+2 i \epsilon\left(e_{\beta} A_{\gamma}\right) Q^{\beta \gamma}{ }_{\zeta \eta}+2 i \epsilon A_{\beta} A_{\gamma} Q^{\beta \gamma}{ }_{\zeta \eta},
$$

where the expression

$$
\nabla_{\zeta} A_{\eta}=e_{\zeta} A_{\eta}-A_{\alpha} \omega^{\alpha}{ }_{\zeta \eta}
$$

denotes the gravity-covariant derivative of the vector $A_{\alpha}$.

An important property of noncommutative spaces with inner derivation-based calculus is the existence of a preferred connection $\theta$,

$$
\theta=-p_{\alpha} \theta^{\alpha} .
$$

The differential can be expressed in the form $d f=-[\theta, f]$. As one can show easily,

$$
d \theta+\theta^{2}=\frac{1}{2 i \epsilon} K_{\alpha \beta} \theta^{\alpha} \theta^{\beta} .
$$

$\theta$ is an example of the Dirac operator in the sense of Connes, [27]. It is invariant under the action of the gauge group: one can see it from

$$
\theta^{\prime}=g^{-1} \theta g+g^{-1} d g
$$

and

$$
g^{-1} \theta g=g^{-1}[\theta, g]+g^{-1} g \theta=-g^{-1} d g+\theta .
$$

The difference between connections $A$ and $\theta, \mathbf{X}=A-\theta$, transforms in the adjoint representation; coefficients $\mathbf{X}_{\alpha}=p_{\alpha}+A_{\alpha}$ are called the covariant coordinates. Expressing the field strength in terms of $\mathrm{X}_{\alpha}$ and the structure elements we obtain

$$
F_{\alpha \beta}=2 P_{\alpha \beta}^{\gamma \delta} \mathrm{X}_{\gamma} \mathrm{X}_{\delta}-F_{\alpha \beta}^{\gamma} \mathrm{X}_{\gamma}-\frac{1}{i \epsilon} K_{\alpha \beta},
$$

that is

$$
F=\mathrm{X}^{2}-\frac{1}{2} F_{\alpha \beta}^{\gamma} \mathrm{X}_{\gamma} \theta^{\alpha} \theta^{\beta}-\frac{1}{2 i \epsilon} K_{\alpha \beta} \theta^{\alpha} \theta^{\beta} .
$$

Covariant coordinates diverge in the commutative limit because $p_{\alpha}$ do (in our case for example, $p_{1}=\frac{i \mu^{2}}{\epsilon} y, p_{2}=-\frac{i \mu^{2}}{\epsilon} x$, etc. so for $\left.\epsilon \rightarrow 0, p_{\alpha} \rightarrow \infty\right)$. For quantization it is thus better to express the action in $A_{\alpha}, F_{\alpha \beta}$ as then we have the control of the commutative limit. On the other hand, if we write the lagrangian in terms of $\mathrm{X}_{\alpha}$ we obtain a polynomial, that is, the kinetic term is absent. The corresponding theory is equivalent to a matrix model, [28, 29], for which the classical equations of motion can often be solved. Covariant coordinates are very useful to keep track of transformation properties under the symmetry group and therefore we will usually write equations parelelly in $A$ and in $\mathrm{X}$.

To distinguish the values of the gauge field on the full three-dimensional truncated Heisenberg space from those defined intrinsically on the two-dimensional Moyal plane, we 
denote the former by $\mathrm{A}_{\alpha}, \mathrm{F}_{\alpha \beta}(\alpha, \beta=1,2,3)$ and the latter by $A_{\alpha}, F_{\alpha \beta}(\alpha, \beta=1,2)$. We will be interested in the subspace $z=0$, where $p_{3}=-\frac{i \mu}{2 \epsilon}, e_{3}=0$. On this subspace the component $\mathrm{A}_{3}$ transforms as a scalar field in the adjoint representation, $\mathrm{A}_{3}^{\prime}=g^{-1} \mathrm{~A}_{3} g$. We denote ${ }^{3}$

$$
\mathrm{A}_{3}=\phi, \quad \mathrm{A}_{1}=A_{1}, \quad \mathrm{~A}_{2}=A_{2}
$$

and equivalently,

$$
\mathrm{X}_{1}=p_{1}+A_{1}, \quad \mathrm{X}_{2}=p_{2}+A_{2}, \quad \mathrm{X}_{3}=-\frac{i \mu}{2 \epsilon}+\phi
$$

On the Moyal plane we would have

$$
\begin{aligned}
D_{\alpha} \phi & =\left[p_{\alpha}+A_{\alpha}, \phi\right]=e_{\alpha} \phi+\left[A_{\alpha}, \phi\right], \\
F_{12} & =e_{1} A_{2}-e_{2} A_{1}+\left[A_{1}, A_{2}\right],
\end{aligned}
$$

while from (3.5) on the truncated Heisenberg space

$$
\begin{aligned}
& \mathrm{F}_{12}=e_{1} \mathrm{~A}_{2}-e_{2} \mathrm{~A}_{1}+\left[\mathrm{A}_{1}, \mathrm{~A}_{2}\right]-\mu \mathrm{A}_{3}, \\
& \mathrm{~F}_{13}=\left[p_{1}+\mathrm{A}_{1}, \mathrm{~A}_{3}\right]-i \epsilon\left\{p_{2}+\mathrm{A}_{2}, \mathrm{~A}_{3}\right\}+2 \mu^{2} \mathrm{~A}_{2} z, \\
& \mathrm{~F}_{23}=\left[p_{2}+\mathrm{A}_{2}, \mathrm{~A}_{3}\right]+i \epsilon\left\{p_{1}+\mathrm{A}_{1}, \mathrm{~A}_{3}\right\}-2 \mu^{2} \mathrm{~A}_{1} z .
\end{aligned}
$$

In particular, for $z=0$ we obtain

$$
\begin{aligned}
& \mathrm{F}_{12}=F_{12}-\mu \phi=\left[\mathrm{X}_{1}, \mathrm{X}_{2}\right]+\frac{i \mu^{2}}{\epsilon}-\mu \phi, \\
& \mathrm{F}_{13}=D_{1} \phi-i \epsilon\left\{p_{2}+A_{2}, \phi\right\}=\left[\mathrm{X}_{1}, \phi\right]-i \epsilon\left\{\mathrm{X}_{2}, \phi\right\}, \\
& \mathrm{F}_{23}=D_{2} \phi+i \epsilon\left\{p_{1}+A_{1}, \phi\right\}=\left[\mathrm{X}_{2}, \phi\right]+i \epsilon\left\{\mathrm{X}_{1}, \phi\right\} .
\end{aligned}
$$

Clearly, on the given subspace vector potential $\mathrm{A}_{\alpha}$ splits into a scalar mode $\phi$ and a vector mode $A_{\alpha}$. We mentioned earlier that the gauge field on the fuzzy sphere behaves similarly, only in that case the scalar component corresponds to the radial degree of freedom $\phi=x^{i} A_{i}+A_{i} x^{i}$ while the remaining two gauge degrees of freedom are tangential, [30]; see also [31-33]. The difference in dimensionsionalities of the basic and the cotangent space, apparently somewhat counterintuitive, seems to follow naturally from the relation which noncommutative geometry has with D-brane physics, [34]. The same kind of effect in the usual Kaluza-Klein reduction one obtains from the assumption that the fields do not depend on the internal that is additional coordinates ( $z$ in our case, $r$ on the sphere).

\section{Yang-Mills action}

We showed that the exterior algebra on the truncated Heisenberg space admits a unique volume form and therefore the integration is well defined. To obtain the Yang-Mills action

\footnotetext{
${ }^{3}$ As we are using antihermitian 1-form $A$, scalar field $\phi$ is also antihermitian; one can obtain real scalar by replacing $\phi \rightarrow i \phi$.
} 
we start from a three-dimensional expression. The third 'integral' will be absorbed implicitly in a rescaling of the coupling constant after reduction to $z=0$. The Yang-Mills action is given by

$$
\mathcal{S}_{Y M}=\frac{1}{16} \operatorname{Tr}\left(\mathrm{F}^{*} \mathrm{~F}+{ }^{*} \mathrm{FF}\right) .
$$

As it was mentioned, we have to symmetrize the product of forms. For $\epsilon=0,(4.1)$ reduces to the standard expression $\mathcal{S}_{Y M}=\frac{1}{4} \operatorname{Tr} \mathrm{F}_{\alpha \beta} \mathrm{F}^{\alpha \beta}$. In our case due to normalization (2.27) we have

$$
\mathcal{S}_{Y M}=\frac{1}{2} \operatorname{Tr}\left(\left(1-\epsilon^{2}\right) \mathrm{F}_{12} \mathrm{~F}^{12}+\mathrm{F}_{13} \mathrm{~F}^{13}+\mathrm{F}_{23} \mathrm{~F}^{23}\right) .
$$

Introducing expressions (3.18) for the first term we obtain

$$
\operatorname{Tr}\left(\mathrm{F}_{12}\right)^{2}=\operatorname{Tr}\left(\left(F_{12}\right)^{2}-2 \mu F_{12} \phi+\mu^{2} \phi^{2}\right)
$$

while the sum of the other two terms after various simplifications becomes

$$
\begin{aligned}
\operatorname{Tr}\left(\left(\mathrm{F}_{13}\right)^{2}+\left(\mathrm{F}_{23}\right)^{2}\right)=\operatorname{Tr} & \left(\left(D_{1} \phi\right)^{2}+\left(D_{2} \phi\right)^{2}+4 \mu^{2} \phi^{2}+4 i \epsilon F_{12} \phi^{2}\right. \\
& \left.-\epsilon^{2}\left\{p_{1}+A_{1}, \phi\right\}^{2}-\epsilon^{2}\left\{p_{2}+A_{2}, \phi\right\}^{2}\right) .
\end{aligned}
$$

Therefore the Yang-Mills action is

$$
\begin{aligned}
\mathcal{S}_{Y M}=\frac{1}{2} \operatorname{Tr} & \left(\left(1-\epsilon^{2}\right)\left(F_{12}\right)^{2}-2\left(1-\epsilon^{2}\right) \mu F_{12} \phi+\left(5-\epsilon^{2}\right) \mu^{2} \phi^{2}+4 i \epsilon F_{12} \phi^{2}\right. \\
& \left.+\left(D_{1} \phi\right)^{2}+\left(D_{2} \phi\right)^{2}-\epsilon^{2}\left\{p_{1}+A_{1}, \phi\right\}^{2}-\epsilon^{2}\left\{p_{2}+A_{2}, \phi\right\}^{2}\right)
\end{aligned}
$$

or using the covariant coordinates,

$$
\begin{aligned}
\mathcal{S}_{Y M}=\frac{1}{2} \operatorname{Tr} & \left(\left(1-\epsilon^{2}\right)\left(\left[\mathrm{X}_{1}, \mathrm{X}_{2}\right]^{2}+\mu^{2} \phi^{2}-\frac{2 i \mu^{3}}{\epsilon} \phi-2 \mu\left[\mathrm{X}_{1}, \mathrm{X}_{2}\right] \phi\right)\right. \\
& \left.+4 i \epsilon\left[\mathrm{X}_{1}, \mathrm{X}_{2}\right] \phi^{2}+\left[\mathrm{X}_{1}, \phi\right]^{2}+\left[\mathrm{X}_{2}, \phi\right]^{2}-\epsilon^{2}\left\{\mathrm{X}_{1}, \phi\right\}^{2}-\epsilon^{2}\left\{\mathrm{X}_{2}, \phi\right\}^{2}\right)
\end{aligned}
$$

The action is obviously gauge invariant.

One observes immediately that for $\epsilon=1$ the kinetic term for the gauge field $F_{12}$ is absent: the action is almost identical to the induced gauge action obtained in $[14,15]$ by the path integration of the scalar field. The difference is in the additional terms which mix $F_{12}$ and $\phi$ : the kinetic term $F_{12} \phi$ and the interaction term $F_{12} \phi^{2}$. Indeed this difference is significant as it changes the vacuum. We can see it from the equations of motion:

$$
\begin{aligned}
\frac{\delta \mathcal{S}_{Y M}}{\delta \phi}=0= & -\left(1-\epsilon^{2}\right) \mu F_{12}+\left(5-\epsilon^{2}\right) \mu^{2} \phi+2 i \epsilon\left\{F_{12}, \phi\right\} \\
& -D^{\alpha} D_{\alpha} \phi-\epsilon^{2}\left\{p^{\alpha}+A^{\alpha},\left\{p_{\alpha}+A_{\alpha}, \phi\right\}\right\}, \\
\frac{\delta \mathcal{S}_{Y M}}{\delta A_{\alpha}}=0= & \left(1-\epsilon^{2}\right) \epsilon^{\alpha \beta} D_{\beta}\left(F_{12}-\mu \phi\right)+2 i \epsilon \epsilon^{\alpha \beta}\left\{D_{\beta} \phi, \phi\right\} \\
& -\left[D_{\alpha} \phi, \phi\right]-\epsilon^{2}\left\{\left\{p^{\alpha}+A^{\alpha}, \phi\right\}, \phi\right\} .
\end{aligned}
$$


It is difficult to solve these equations in the most general case. Confining to constant solutions we obtain two,

$$
A_{1}=0, A_{2}=0, \phi=0 \quad \text { and } \quad \mathrm{X}_{1}=0, \mathrm{X}_{2}=0, \phi=\frac{i \mu}{\epsilon} .
$$

Obviously the first solution is the usual vacuum which can be used for quantization. The second solution $A_{1}=-\frac{i \mu^{2}}{\epsilon} y, A_{2}=\frac{i \mu^{2}}{\epsilon} x, \phi=\frac{i \mu}{\epsilon}$ has a constant field strength $F_{12}=\frac{i \mu^{2}}{\epsilon}$ and therefore larger, nonzero energy.

\section{Chern-Simons action}

It is not completely straightforward to deduce what would in general be the noncommutative equivalent of the usual Chern-Simons action, [35-37]. Clearly it has to be an expression which is, up to surface terms, invariant under the gauge group

$$
\delta \mathcal{S}_{C S}=\delta \operatorname{Tr} L_{C S}=0 .
$$

One would impose further the correct commutative limit. However, not in all cases a topological definition would be appropriate because considerations which include surface terms are in the case of finite matrix spaces vacuous: these spaces do not have boundary (that is, $\operatorname{Tr} d f$ is always zero because $d f$ is expressed in terms of commutators). Also, it is not clear that it is in general possible to solve Equation (5.1) and find the current

$$
\delta L_{C S}=d J
$$

as in the commutative case. Some kind of 'canonical' differential calculus, analogous to the de Rham calculus and defined in any number of dimensions does not exist for noncommutative spaces; therefore in formulae it is not justified a priori to pass from space of one dimension to the space of another.

In [38], as generalization of the Chern-Simons action for the space with constant noncommutativity Polychronakos proposed the following action

$$
\mathcal{S}_{C S}=\alpha \operatorname{Tr} \mathbf{X}^{2 n+1}
$$

where $\mathbf{X}$ is the covariant coordinate 1 -form, $\mathbf{X}=\mathbf{X}_{\alpha} \theta^{\alpha}$. Clearly, as $\mathbf{X}$ transforms in the adjoint representation, (5.3) is invariant under the gauge group and contains terms of the correct order, for example the usual $F^{n} A$. Moreover, (5.3) has a reasonable commutative limit: the limit of the noncommutative $U_{1}$ action (5.3) is the nonabelian $U_{n}$ Chern-Simons action defined on commutative space, [38].

The advantage of action $\operatorname{Tr} \mathrm{X}^{3}$ in our framework is that it explicitly symmetrizes the factors of the volume 3-form $\Theta$ which are otherwise not cyclic. We will therefore use (5.3) as a definition of the Chern-Simons action and explore its implications for the truncated Heisenberg space. Applying the projector $\Delta_{\zeta \eta \xi}^{\alpha \beta \gamma}$ we have

$$
\operatorname{Tr} \mathbf{X}^{3}=\operatorname{Tr}\left(\mathbf{X}_{\alpha} \mathbf{X}_{\beta} \mathbf{X}_{\gamma} \Delta_{\zeta \eta \xi}^{\alpha \beta \gamma} \theta^{\zeta} \theta^{\eta} \theta^{\xi}\right)
$$


and therefore we obtain, in components,

$$
\mathcal{S}_{C S}=\frac{\alpha \mu}{3} \operatorname{Tr}\left(\left(3-\epsilon^{2}\right)\left[\mathrm{X}_{1}, \mathbf{X}_{2}\right] \mathbf{X}_{3}+2 i \epsilon\left(\mathrm{X}_{1}^{2}+\mathrm{X}_{2}^{2}\right) \mathbf{X}_{3}\right) .
$$

Equivalently in terms of the gauge potentials,

$$
\mathcal{S}_{C S}=\frac{\alpha \mu}{3} \operatorname{Tr}\left(\left(3-\epsilon^{2}\right)\left(F_{12}-\frac{i \mu^{2}}{\epsilon}\right) \phi+\frac{2 i \epsilon}{3}\left(\left(p_{1}+A_{1}\right)^{2}+\left(p_{2}+A_{2}\right)^{2}\right)\left(\phi-\frac{i \mu}{2 \epsilon}\right)\right) .
$$

The Chern-Simons action also depends on coordinates. Its variations are

$$
\begin{aligned}
& \frac{\delta \mathcal{S}_{C S}}{\delta \phi}=\frac{\alpha \mu}{3}\left(\left(3-\epsilon^{2}\right)\left(F_{12}-\frac{i \mu^{2}}{\epsilon}\right)+2 i \epsilon\left(\left(p_{1}+A_{1}\right)^{2}+\left(p_{2}+A_{2}\right)^{2}\right)\right), \\
& \frac{\delta \mathcal{S}_{C S}}{\delta A_{\alpha}}=\frac{\alpha \mu}{3}\left(\left(3-\epsilon^{2}\right) \epsilon^{\alpha \beta} D_{\beta} \phi+2 i \epsilon\left\{p_{\alpha}+A_{\alpha}, \phi-\frac{i \mu}{2 \epsilon}\right\}\right) .
\end{aligned}
$$

The equations which correspond to the pure Chern-Simons action have one constant solution, $\mathrm{X}_{1}=0, \mathrm{X}_{2}=0, \phi=\frac{i \mu}{\epsilon}$. The other vacuum $A_{1}=0, A_{2}=0, \phi=0$ of (4.5)-(4.6) is absent in general. The sum $\mathcal{S}_{Y M}+\mathcal{S}_{C S}$ however has interesting properties: by an appropriate choice of coefficient $\alpha$, the mixed term $F_{12} \phi$ (which is potentially difficult for quantization) can be cancelled. Furthermore, the sum of the two actions has constant solution $A_{1}=0, A_{2}=0, \phi=i \mu$ (describing the spontaneous symmetry breaking) for a particular choice of parameters $\epsilon=1, \alpha=6$. Whether one should include $\mathcal{S}_{C S}$ in the gauge field action or not is not completely clear; defining the BRST complex we shall proceed with $\mathcal{S}_{Y M}$ only, the inclusion of $\mathcal{S}_{C S}$ being straightforward.

\section{$6 \quad$ BRST invariance}

The gauge fixing of the Yang-Mills action can be done straightforwardly. The simplest choice is the Lorentz gauge,

$$
\mathcal{G}=e_{\alpha} A^{\alpha}=\partial_{1} A^{1}+\partial_{2} A^{2}
$$

According to the usual procedure the quantum action is given by

$$
\mathcal{S}=\mathcal{S}_{Y M}+\mathcal{S}_{g f}
$$

with

$$
\mathcal{S}_{g f}=\operatorname{Tr}\left(B e_{\alpha} A^{\alpha}+\frac{\alpha}{2} B B-\bar{c} e_{\alpha} D^{\alpha} c\right)
$$

where we introduced the ghost $c$, the antighost $\bar{c}$ and the auxilliary field $B$. The BRST transformation $s$ acting on the gauge potential can be defined as

$$
s A_{\alpha}=D_{\alpha} c=e_{\alpha} c+i\left[A_{\alpha}, c\right] .
$$

$F_{\alpha \beta}, \phi$ and $\mathrm{X}_{\alpha}=p_{\alpha}+A_{\alpha}$ transform in the adjoint representation and therefore we have

$$
\begin{aligned}
s F_{\alpha \beta} & =\left[F_{\alpha \beta}, c\right], \\
s \phi & =[\phi, c], \\
s \mathrm{X}_{\alpha} & =\left[\mathrm{X}_{\alpha}, c\right]=e_{\alpha} c+i\left[A_{\alpha}, c\right]=s A_{\alpha} .
\end{aligned}
$$


This means also that the momenta $p_{\alpha}$ are BRST-invariant, $s p_{\alpha}=0$. Of course the Leibniz rule for $s$ holds, for example

$$
s\left\{\mathrm{X}_{\alpha}, \phi\right\}=\left\{\left[\mathrm{X}_{\alpha}, c\right], \phi\right\}+\left\{\mathrm{X}_{\alpha},[\phi, c]\right\}=\left[\left\{\mathrm{X}_{\alpha}, \phi\right\}, c\right] .
$$

For the ghosts and the auxiliary field we can also define the BRST transformation as usual

$$
s c=-c^{2}, \quad s \bar{c}=B, \quad s B=0 .
$$

With these definitions it is easy to check that $s$ is nilpotent, $s^{2}=0$, and then also that $s \mathcal{S}_{g f}=0$ as the gauge-fixing term is equal to

$$
\mathcal{S}_{g f}=\operatorname{Tr} s\left(\bar{c} e_{\alpha} A^{\alpha}+\frac{\alpha}{2} \bar{c} B\right)
$$

For the classical Yang-Mills part of the action $s \mathcal{S}_{Y M}=0$ because of its gauge invariance.

It is quite clear that the gauge fixing (6.1) is a possible gauge choice. However as we are in the curved space, one might wonder whether a more natural choice is the covariant gauge, $\nabla_{\alpha} A^{\alpha}$. The covariant derivative was defined already in (3.7); we denote

$$
\mathcal{F}=e_{\alpha} A^{\alpha}+A^{\beta} \omega^{\alpha}{ }_{\alpha \beta} .
$$

To calculate $\mathcal{F}$ we need the linear connection. Asssuming that $\omega_{\alpha \beta \gamma}=\frac{1}{2}\left(C_{\alpha \beta \gamma}-C_{\beta \gamma \alpha}+\right.$ $\left.C_{\gamma \alpha \beta}\right)$ as in [20], we obtain

$$
\omega^{\alpha}{ }_{\alpha 1}=-2 \mu^{2} x, \quad \omega^{\alpha}{ }_{\alpha 2}=-2 \mu^{2} y, \quad \omega^{\alpha}{ }_{\alpha 3}=0,
$$

and therefore

$$
\mathcal{F}=\partial_{1} A_{1}+\partial_{2} A_{2}-2 \mu^{2} A_{1} x-2 \mu^{2} A_{2} y .
$$

As $\mathcal{F}$ is not hermitian the possible gauge choices are $\mathcal{F} \mathcal{F}^{\dagger}$ or $\frac{1}{4}\left(\mathcal{F}+\mathcal{F}^{\dagger}\right)^{2}$. In both cases the procedure to introduce $\mathcal{S}_{g f}$ and prove the BRST invariance is straightforward, only the corresponding expressions are somewhat longer. To the comparative advantages and shortcomings of different gauge choices we shall return in our future work.

\section{Conclusions}

The aim of the paper was to derive the action for the $U_{1}$ gauge field on the truncated Heisenberg algebra and to reduce it to the Moyal subspace, in order to obtain a candidate for renormalizable noncommutative gauge theory. Various other proposals were discussed in the literature, $[14,15,18,19,41]$. Our main idea was to apply the geometric logic developed in [20]: it was shown there that renormalizability of the Grosse-Wulkenhaar action can be attributed to the interaction with the background curvature. If indeed geometric properties of the background space play a role, a similar result is to be expected for the gauge and other fields. An alternative way to understand how renormalizability can be related to a noncommutative geometry is the fact that the underlying space in question is an algebra 
of finite matrices. Defining the scalar or the gauge fields on this algebra provides therefore a geometrically consistent way to define matrix regularization.

The truncated Heisenberg algebra is a three-dimensional noncommutative space. Properties of its cotangent space were discussed in [20]; here in order to define gauge fields we explore the structure of the spaces of 2-forms and 3-forms. This enables to define the Hodge-dual and the volume form and consequently to obtain the Yang-Mills action. When written in frame components the gauge potentials couple to the connection, which results in an explicit coordinate dependence of the lagrangian. This is the property we aimed to obtain, as in the case of the scalar field the coordinate dependence modified the usual propagator to the Mehler kernel and that had ensured the absence of the UV/IR mixing and eventually, the renormalizability.

To get a theory in two dimensions in the second step of the construction we constrain to the subspace $z=0$; this subspace is algebraically but not geometrically equivalent to the Moyal space. The degrees of freedom of the vector potential $\mathrm{A}_{\alpha}, \alpha=1,2,3$ split then into a scalar field $\phi$ and a two-dimensional gauge field $A_{\alpha}, \alpha=1,2 . \phi$ and $A_{\alpha}$ are coupled; the coupling is completely fixed because it comes from a higher-dimensional Yang-Mills action. It is possible to write the resulting lagrangian in terms of the covariant coordinates also. The corresponding classical equations of motion have a solution $\phi=0, A_{\alpha}=0$ suitable as a vacuum for the quantization. We also discuss the Chern-Simons action and show how it changes the vacuum. Finally, we introduce the appropriate gauge fixing and prove the BRST invariance of the gauge-fixed action.

The coordinate dependence of the lagrangian was in some previous works attributed to the external electromagnetic field, [10]; here we relate it to the background gravity. Note that there is a further possibility to include coordinates covariantly, which is due to a special form of the differential calculus, $d f=-\left[p_{\alpha}, f\right] \theta^{\alpha}=-[\theta, f]$. The coordinatedependent quantities $\mathrm{X}_{\alpha}=\mathrm{A}_{\alpha}+p_{\alpha}$ in this case transform in the adjoint representation of the gauge group and obviously they can be included in the action invariantly, [26].

Let us elaborate on relations between the gravitational and the gauge fields on noncommutative spaces a bit further. It has recently been noticed [39] that one can interpret noncommutative gravity as an induced theory obtained by summing over a set of matrix models of electromagnetism in a way which is reminiscent of the 'induction' of (euclidean) gravity [40] by summing over all the quantum fluctuations of a scalar field. One can give a 'derivation' of this result in three steps. One first notices that the Dirac operator $\theta$ can be interpreted as an electromagnetic potential. One then recalls that this same operator determines the differential calculus. Finally one reiterates the argument that the differential calculi over an algebra stand in one to one correspondence with the metrics consistent with the algebra. Some details of the first step are given in section 3; a discussion of the other steps can be found in the literature, [21].

To summarize: we obtained a geometric action which contains besides the gauge field a scalar. Their coupling is of a particular form and in fact the whole construction has similarities with the Kaluza-Klein reduction, [43]. In principle, this coupling can induce cancellation of divergences in the quantization: we plan to analyze in more details quantization and renormalization of the proposed model in our future work. 


\section{Acknowledgments}

This work was supported by ESF grants 2747 and 2749 through the Quantum Gravity Network and by 141036 project of MNTR, Serbia.

Open Access. This article is distributed under the terms of the Creative Commons Attribution Noncommercial License which permits any noncommercial use, distribution, and reproduction in any medium, provided the original author(s) and source are credited.

\section{References}

[1] M. Dubois-Violette, J. Madore and R. Kerner, Gauge bosons in a noncommutative geometry, Phys. Lett. B 217 (1989) 485 [SPIRES].

[2] M. Dubois-Violette, J. Madore and R. Kerner, Classical bosons in a noncommutative geometry, Class. Quant. Grav. 6 (1989) 1709 [SPIRES].

[3] M. Dubois-Violette, R. Kerner and J. Madore, Noncommutative Differential Geometry and New Models of Gauge Theory, J. Math. Phys. 31 (1990) 323 [SPIRES].

[4] N. Seiberg and E. Witten, String theory and noncommutative geometry, JHEP 09 (1999) 032 [hep-th/9908142] [SPIRES].

[5] I. Chepelev and R. Roiban, Convergence theorem for non-commutative Feynman graphs and renormalization, JHEP 03 (2001) 001 [hep-th/0008090] [SPIRES].

[6] I. Chepelev and R. Roiban, Renormalization of quantum field theories on noncommutative $R^{d}$. I: Scalars, JHEP 05 (2000) 037 [hep-th/9911098] [SPIRES].

[7] H. Grosse and R. Wulkenhaar, Renormalisation of $\phi^{4}$ theory on noncommutative $R^{2}$ in the matrix base, JHEP 12 (2003) 019 [hep-th/0307017] [SPIRES].

[8] H. Grosse and R. Wulkenhaar, Renormalisation of $\phi^{4}$ theory on noncommutative $R^{4}$ in the matrix base, Commun. Math. Phys. 256 (2005) 305 [hep-th/0401128] [SPIRES].

[9] H. Grosse and R. Wulkenhaar, Renormalisation of $\phi^{4}$-theory on non-commutative $R^{4}$ to all orders, Lett. Math. Phys. 71 (2005) 13 [SPIRES].

[10] E. Langmann and R.J. Szabo, Duality in scalar field theory on noncommutative phase spaces, Phys. Lett. B 533 (2002) 168 [hep-th/0202039] [SPIRES].

[11] R. Gurau, J. Magnen, V. Rivasseau and A. Tanasa, A translation-invariant renormalizable non-commutative scalar model, Commun. Math. Phys. 287 (2009) 275 [arXiv:0802.0791] [SPIRES].

[12] R. Gurau, J. Magnen, V. Rivasseau and F. Vignes-Tourneret, Renormalization of non-commutative $\phi_{4}^{4}$ field theory in $x$ space, Commun. Math. Phys. 267 (2006) 515 [hep-th/0512271] [SPIRES].

[13] D.N. Blaschke et al., On the Problem of Renormalizability in Non-Commutative Gauge Field Models. A Critical Review, Fortschr. Phys. 58 (2010) 364 [arXiv:0908.0467] [SPIRES].

[14] A. de Goursac, J.C. Wallet and R. Wulkenhaar, Noncommutative induced gauge theory, Eur. Phys. J. C 51 (2007) 977 [hep-th/0703075] [SPIRES].

[15] H. Grosse and M. Wohlgenannt, Induced Gauge Theory on a Noncommutative Space, Eur. Phys. J. C 52 (2007) 435 [hep-th/0703169] [SPIRES]. 
[16] A. de Goursac, J.-C. Wallet and R. Wulkenhaar, On the vacuum states for noncommutative gauge theory, Eur. Phys. J. C 56 (2008) 293 [arXiv:0803.3035] [SPIRES].

[17] H. Grosse and R. Wulkenhaar, 8D-spectral triple on 4 D-Moyal space and the vacuum of noncommutative gauge theory, arXiv:0709.0095 [SPIRES].

[18] D.N. Blaschke, H. Grosse and M. Schweda, Non-commutative U(1) gauge theory on $R^{4}(\Theta)$ with oscillator term and BRST symmetry, Europhys. Lett. 79 (2007) 61002 [arXiv:0705.4205] [SPIRES].

[19] D.N. Blaschke, A. Rofner, M. Schweda and R.I.P. Sedmik, Improved Localization of a Renormalizable Non-Commutative Translation Invariant U(1) Gauge Model, Europhys. Lett. 86 (2009) 51002 [arXiv:0903.4811] [SPIRES].

[20] M. Burić and M. Wohlgenannt, Geometry of the Grosse-Wulkenhaar Model, JHEP 03 (2010) 053 [arXiv:0902.3408] [SPIRES].

[21] J. Madore, An introduction to noncommutative differential geometry and itsphysical applications, Lond. Math. Soc. Lect. Note Ser. 257 (2000) 1 [SPIRES].

[22] J. Madore, The fuzzy sphere, Class. Quant. Grav. 9 (1992) 69 [SPIRES].

[23] A. Sitarz, Noncommutative differential calculus on the kappa Minkowski space, Phys. Lett. B 349 (1995) 42 [hep-th/9409014] [SPIRES].

[24] G. Amelino-Camelia, A. Marciano and D. Pranzetti, On the 5D differential calculus and translation transformations in $4 D$ kappa-Minkowski noncommutative spacetime, Int. J. Mod. Phys. A 24 (2009) 5445 [arXiv:0709.2063] [SPIRES].

[25] G. Fiore and J. Madore, Leibniz Rules and Reality Conditions, [math/9806071].

[26] E. Cagnache, T. Masson and J.-C. Wallet, Noncommutative Yang-Mills-Higgs actions from derivation- based differential calculus, arXiv:0804.3061 [SPIRES].

[27] A. Connes, Noncommutative Geometry, Academic Press (1994).

[28] H. Aoki et al., Noncommutative Yang-Mills in IIB matrix model, Nucl. Phys. B 565 (2000) 176 [hep-th/9908141] [SPIRES].

[29] J. Ambjørn, Y.M. Makeenko, J. Nishimura and R.J. Szabo, Finite $N$ matrix models of noncommutative gauge theory, JHEP 11 (1999) 029 [hep-th/9911041] [SPIRES].

[30] H. Grosse and J. Madore, A Noncommutative version of the Schwinger model, Phys. Lett. B 283 (1992) 218 [SPIRES].

[31] H. Steinacker, Quantized gauge theory on the fuzzy sphere as random matrix model, Nucl. Phys. B 679 (2004) 66 [hep-th/0307075] [SPIRES].

[32] S. Iso, Y. Kimura, K. Tanaka and K. Wakatsuki, Noncommutative gauge theory on fuzzy sphere from matrix model, Nucl. Phys. B 604 (2001) 121 [hep-th/0101102] [SPIRES].

[33] Y. Kimura, Noncommutative gauge theories on fuzzy sphere and fuzzy torus from matrix model, Prog. Theor. Phys. 106 (2001) 445 [hep-th/0103192] [SPIRES].

[34] A.Y. Alekseev, A. Recknagel and V. Schomerus, Brane dynamics in background fluxes and non-commutative geometry, JHEP 05 (2000) 010 [hep-th/0003187] [SPIRES].

[35] A.H. Chamseddine and J. Fröhlich, The Chern-Simons action in noncommutative geometry, J. Math. Phys. 35 (1994) 5195 [hep-th/9406013] [SPIRES]. 
[36] C.-S. Chu, Induced Chern-Simons and WZW action in noncommutative spacetime, Nucl. Phys. B 580 (2000) 352 [hep-th/0003007] [SPIRES].

[37] N.E. Grandi and G.A. Silva, Chern-Simons action in noncommutative space, Phys. Lett. B 507 (2001) 345 [hep-th/0010113] [SPIRES].

[38] A.P. Polychronakos, Noncommutative Chern-Simons terms and the noncommutative vacuum, JHEP 11 (2000) 008 [hep-th/0010264] [SPIRES].

[39] H. Steinacker, Emergent Gravity from Noncommutative Gauge Theory, JHEP 12 (2007) 049 [arXiv: 0708.2426] [SPIRES].

[40] A. D. Sakharov, Vacuum quantum fluctuations in curved space and the theory of gravitation, Sov. Phys. Dokl. 12 (1968) 1040 [Dokl. Akad. Nauk Ser. Fiz. 177 (1967) 70] [Sov. Phys. Usp. 34 (1991) 394].

[41] D.N. Blaschke, A. Rofner, R.I.P. Sedmik and M. Wohlgenannt, On Non-Commutative $U^{*}(1)$ Gauge Models and Renormalizability, arXiv:0912.2634 [SPIRES].

[42] D.N. Blaschke, H. Grosse, E. Kronberger, M. Schweda and M. Wohlgenannt, Loop Calculations for the Non-Commutative U(1) Gauge Field Model with Oscillator Term, Eur. Phys. J. C 67 (2010) 575 [arXiv:0912.3642] [SPIRES].

[43] J. Madore, Kaluza-Klein aspects of noncommutative geometry, in Differential Geometric Methods in Theoretical Physics, A.I. Solomon ed., World Scientific Publishing, Chester U.K. (1988), pp. 243-252. 